\title{
An overview of Suomi NPP VIIRS calibration maneuvers
}

\author{
James J. Butler ${ }^{\mathrm{a} *}$, Xiaoxiong Xiong ${ }^{\mathrm{a}}$, Robert A. Barnes ${ }^{\mathrm{b}}$, Frederick S. Patt ${ }^{\mathrm{b}}$, \\ Junqiang Sun ${ }^{\mathrm{C}}$, and Kwofu Chiang ${ }^{\mathrm{C}}$ \\ ${ }^{\mathrm{a}}$ NASA Goddard Space Flight Center, Greenbelt, MD 20661 \\ b Science Applications International Corporation, Beltsville, MD 20605 \\ 'Sigma Space Corporation, Lanham MD 0606
}

\begin{abstract}
The first Visible Infrared Imager Radiometer Suite (VIIRS) instrument was successfully launched on-board the Suomi National Polar-orbiting Partnership (SNPP) spacecraft on October 28, 2011. Suomi NPP VIIRS observations are made in 22 spectral bands, from the visible (VIS) to the long-wave infrared (LWIR), and are used to produce 22 Environmental Data Records (EDRs) with a broad range of scientific applications. The quality of these VIIRS EDRs strongly depends on the quality of its calibrated and geo-located Sensor Date Records (SDRs). Built with a strong heritage to the NASA's EOS MODerate resolution Imaging Spectroradiometer (MODIS) instrument, the VIIRS is calibrated on-orbit using a similar set of on-board calibrators (OBC), including a solar diffuser (SD) and solar diffuser stability monitor (SDSM) system for the reflective solar bands (RSB) and a blackbody (BB) for the thermal emissive bands (TEB). Onorbit maneuvers of the SNPP spacecraft provide additional calibration and characterization data from the VIIRS instrument which cannot be obtained pre-launch and are required to produce the highest quality SDRs. These include multiorbit yaw maneuvers for the characterization of SD and SDSM screen transmission, quasi-monthly roll maneuvers to acquire lunar observations to track sensor degradation in the visible through shortwave infrared, and a driven pitch-over maneuver to acquire multiple scans of deep space to determine TEB response versus scan angle (RVS). This paper provides an overview of these three SNPP calibration maneuvers. Discussions are focused on their potential calibration and science benefits, pre-launch planning activities, and on-orbit scheduling and implementation strategies. Results from calibration maneuvers performed during the Intensive Calibration and Validation (ICV) period for the VIIRS sensor are illustrated. Also presented in this paper are lessons learned regarding the implementation of calibration spacecraft maneuvers on follow-on missions.
\end{abstract}

\section{Keywords: VIIRS, SNPP, maneuvers, on-orbit calibration, characterization}

\section{INTRODUCTION}

The majority of planned low Earth orbiting remote sensing spacecraft maneuvers are performed in support of orbital adjustment and maintenance or collision avoidance activities. These maneuvers are primarily executed using on-orbit propellant burns and result in a physical change in the spacecraft orbit. A very small fraction of planned spacecraft maneuvers has been performed in support of the radiometric calibration and characterization of on-board optical instruments. These calibration and characterization maneuvers have been performed using spacecraft reaction wheels to minimize instrument contamination and do not affect the spacecraft orbit. From 1996 to 2010, the OrbView-2 spacecraft executed monthly backward pitch over maneuvers on the dark side of the orbit to enable the Sea-viewing Wide Field-ofView (SeaWiFS) instrument to view the Moon ${ }^{1}$. On February 2 and 6 and April 6, 2001, the Earth Observation-1 spacecraft was maneuvered using a series of pitch and roll maneuvers to enable the Hyperion instrument to acquire raster data

*(JJB, contact author) James.J.Butler@nasa.gov; phone (301) 614-5942; Earth Sciences Division, Biospheric Sciences Laboratory, Code 618, NASA Goddard Space Flight Center, Greenbelt, MD, USA 20661 
of the Moon ${ }^{2}$. The NASA Terra and Aqua spacecraft have performed a large number of maneuvers in support of instrument calibration and characterization since 2000 and 2002, respectively. The Terra spacecraft has performed 116 roll maneuvers to enable the Moderate Resolution Imaging Spectroradiometer (MODIS) Terra instrument to acquire full disc images of the Moon at a lunar phase of $+55^{\circ}$. Likewise, the Aqua spacecraft has performed 95 roll maneuvers to enable the MODIS Aqua instrument to acquire full disc images of the $\mathrm{Moon}^{3}$ at a lunar phase of $-55^{\circ}$. These lunar images are integrated, and the resulting integrated irradiances for each MODIS band are compared to the output of the USGS lunar irradiance model. These data are ultimately used in trending degradation in the spectral radiometric responsivities of the two MODIS instruments. Images of the Moon acquired by MODIS have also been used to characterize instrument on-orbit spatial performance ${ }^{4}$. On April 25 and 26, 2000, December 5 and 11, 2002, and January 15 and 16, 2003, the Terra spacecraft performed a series of multi-angle, multi-orbit yaw maneuvers corresponding to the range of solar incident angles experienced by the MODIS Terra instrument during its on-board solar diffuser calibrations ${ }^{5,6}$ over the Terra mission. Similarly, series of multi-angle, multi-orbit yaw maneuvers were performed by the Aqua spacecraft on June 14, 15, 23, and 24, 2002 in support of the characterization of the performance of the MODIS Aqua solar diffuser calibration system. On March 26 and April 14, 2003, the Terra satellite successfully performed a backward pitch over maneuver to enable the MODIS instrument to determine response versus scan angle in its thermal bands, the ASTER instrument to directly determine the dc offset in its thermal bands, and the CERES instrument to determine its dc signal offsets. Since the April 14 pitch over maneuver included a view of the Moon, the MODIS, the Multi-angle Imaging SpectroRadiometer (MISR), and ASTER instruments were also able to utilize the Moon as a common, on-orbit crosscomparison target. Lastly, on August 10, 2006, the NOAA-14 spacecraft was commanded into an inertial hold to enable the Microwave Sounding Unit (MSU) to scan deep space and to characterize any cross-track asymmetry in its antenna response ${ }^{7}$.

On October 28, 2011, the Suomi NPP satellite containing five Earth observing instruments was successfully launched on a Delta-2 rocket from Vandenburg Air Force Base in California. The five instruments on-board SNPP included the Visible Infrared Imaging Radiometer Suite (VIIRS), the Advanced Technology Microwave Sounder (ATMS), the Ozone Mapping and Profiling Suite (OMPS), the Cross-track Infrared Sounder (CrIS), and the Clouds and the Earth's Radiance Energy System (CERES) instrument. The SNPP is a multi-agency mission with requirements to provide risk reduction for future NOAA operational missions and to provide research data continuity for the NASA Earth Observing System (EOS) missions. A key part of the meeting latter requirement is the ability of the SNPP instruments to produce data of sufficiently high quality to be used in climate studies and to continue climate data records.

Each SNPP instrument underwent extensive, dedicated, pre-launch calibration and characterization testing at the instrument level and at the observatory level following instrument integration onto the SNPP spacecraft. However, prelaunch calibration and characterization had its limitations on what could be accomplished with respect to the golden rule of testing remote sensing instruments "as they fly." That is, certain instrument calibration and characterization measurements which for reasons of cost, schedule, and difficulty are more accurately performed in the on-orbit environment. In addition, tracking the long-term radiometric performance of the instrument requires careful planning and precise execution of an operational calibration strategy, including calibration maneuvers. These measurements required precise spacecraft maneuvers to enable instruments to acquire or scan targets of radiometric interest. Since launch, the SNPP spacecraft has been maneuvered 36 times in support of the calibration and characterization of its on-board remote sensing instruments. Of those maneuvers, 22 have been dedicated to understanding, improving, and completing the calibration and characterization specifically of the SNPP VIIRS instrument. These VIIRS-specific spacecraft maneuvers included the following:

- 6 rolls of less than $-14^{\circ}$ to enable the VIIRS to acquire images of a $51^{\circ}$ to $55^{\circ}$ phase Moon to track instrument radiometric degradation in the visible, near infrared, and shortwave infrared bands;

- 15 yaw maneuvers over a range of $-6^{\circ}$ to $10.9^{\circ}$ to perform a seasonal mapping of the radiometric performance of the VIIRS on-board solar diffuser and solar diffuser stability monitor calibration system;

- and a complete pitch-over of the SNPP spacecraft on the dark side of the orbit to enable multiple scans of deep space to characterize the thermal infrared response versus scan angle of the VIIRS instrument.

This paper describes each of the SNPP VIIRS maneuvers, presents their scientific rationale, and presents the acquired data and results. The paper concludes with lessons learned for implementing spacecraft maneuvers in support of remote sensing instrument calibration and characterization on future missions. 


\section{SPACECRAFT MANEUVERS AND RESULTS}

\section{2a. Spacecraft Roll Maneuvers}

While MODIS views the Moon through its spaceview port $^{8}$, VIIRS views the Moon through its space view (SV) sector which is located at the beginning of the VIIRS earth view port. For VIIRS, the SV is scanned before the start of the Earth view, between $66.1^{\circ}$ and $65.25^{\circ}$ from nadir. At the SNPP altitude of $828 \mathrm{~km}$, the Earth limb is about $62^{\circ}$ from nadir, so the SV is about $3^{\circ}$ above the limb at the nominal spacecraft attitude. As the spacecraft moves about the Earth in orbit, the SV will scan a conical trajectory on the sky, centered on a point that is opposite the orbit angular momentum vector, or orbit normal. For the current SNPP orbit (1:24 PM ascending node, 98.6 inclination) the negative orbit normal has a celestial latitude of $8.6^{\circ}$ and a celestial longitude equivalent to a $69^{\circ}$ lunar phase. The lunar views occur when the Moon passes through the SV trajectory. This geometry is illustrated in Fig. 1, and a representative VIIRS on-orbit lunar roll maneuver is shown in Fig. 2.

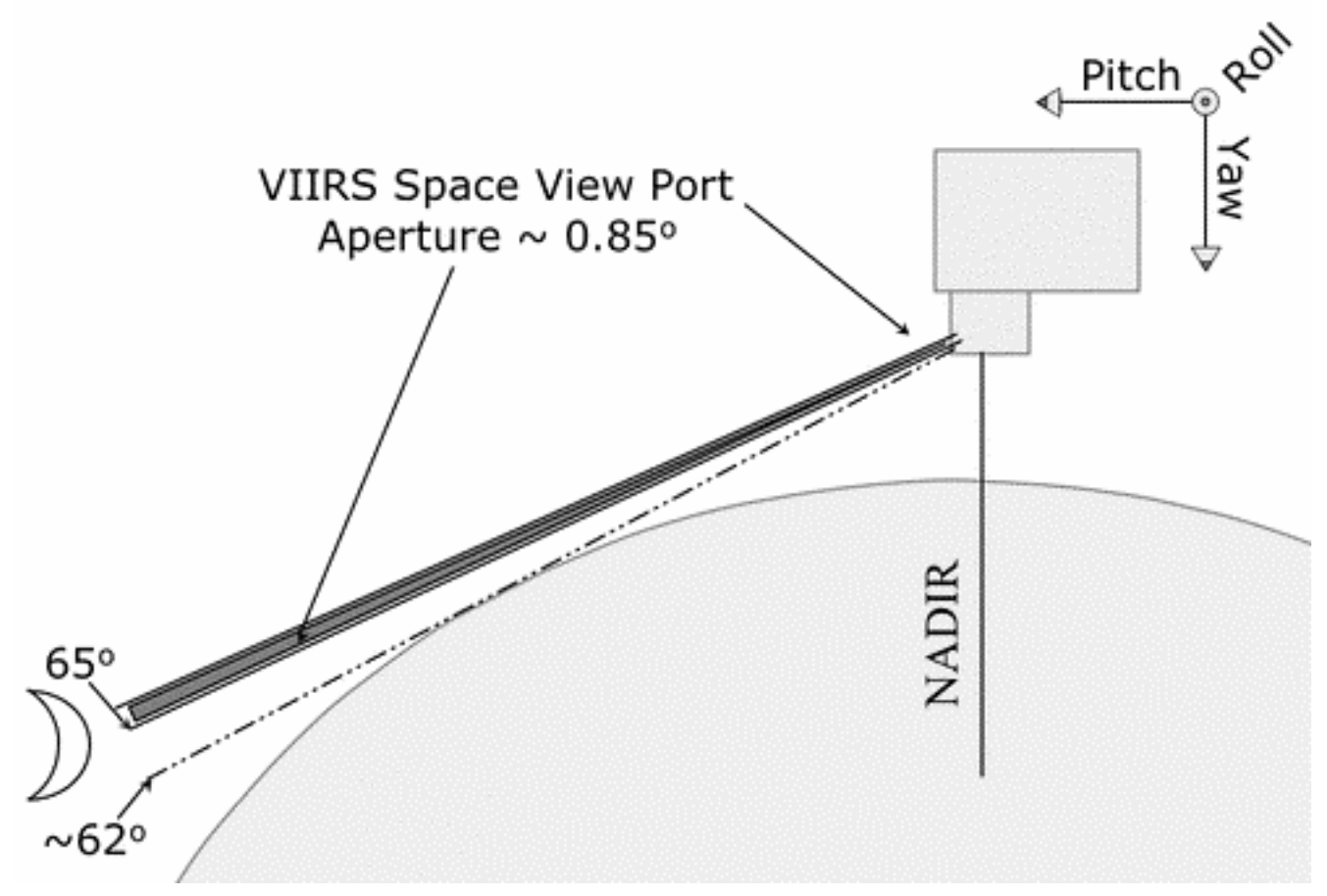

Figure 1. VIIRS space view port geometry

To analyze a typical set of VIIRS lunar views, we computed the Moon's position for every day in 2012 (the first full year of SNPP on-orbit operation), along with the corresponding phase and celestial latitude. The celestial latitude vs. phase is shown in Fig. 3a for the first half of 2012, and in Fig. 3b for the second half. The figures also show the SV trajectory on the sky as concentric circles. The lunar views occur when the Moon's position falls between the two circles. In most months there are two views, as the Moon's trajectory crosses that of the SV twice. The range of phase angles for all views is about $44^{\circ}$ to $93^{\circ}$.

Fig. 3a shows that during the first six months, clear lunar views are available in every month. Fig. 3b shows that, in the second half of the year, clear lunar views are available only during the last three months, and there is one marginal view at a phase of about $62^{\circ}$ (triangles). During the other three months, the lunar trajectory passes well outside of the SV. Note that for the SV geometry (Fig. 1) this also places the Moon below the Earth limb in these months. Thus, in 2012 there are 9 clear views and one marginal view. These conditions will be essentially unchanged in other years, and therefore eight or nine lunar views are available each year. 


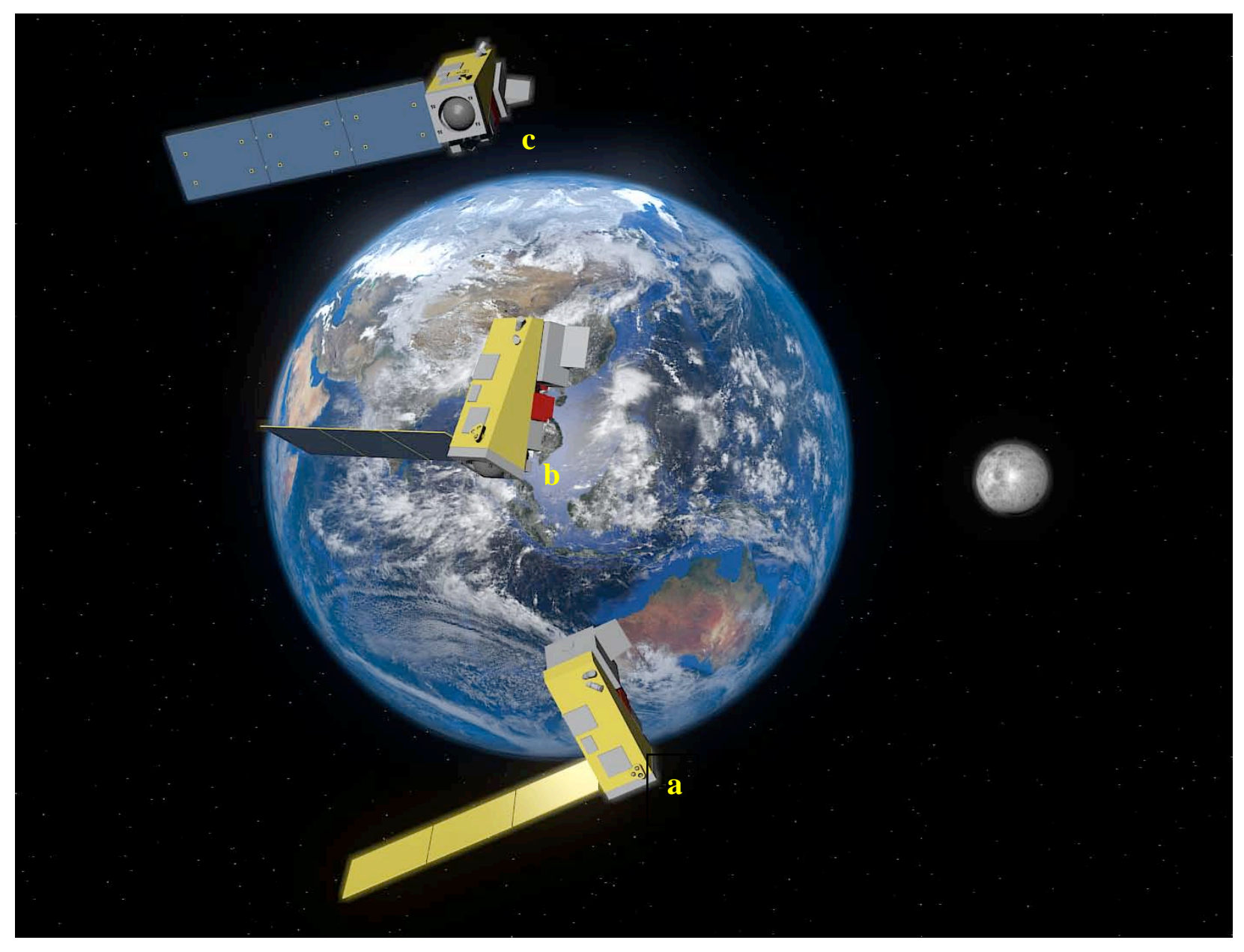

Figure 2. The SNPP spacecraft roll maneuver to enable VIIRS to view the Moon in its reflected solar bands.

a. The SNPP spacecraft is in its nominal Earth viewing mode after it has emerged from the dark side of the orbit after passing over the South Pole.

b. The SNPP spacecraft is rolled to an angle $\leq-14^{\circ}$ enabling the VIIRS instrument to view the Moon.

c. The SNPP spacecraft after it has been rolled back to nominal Earth viewing mode.
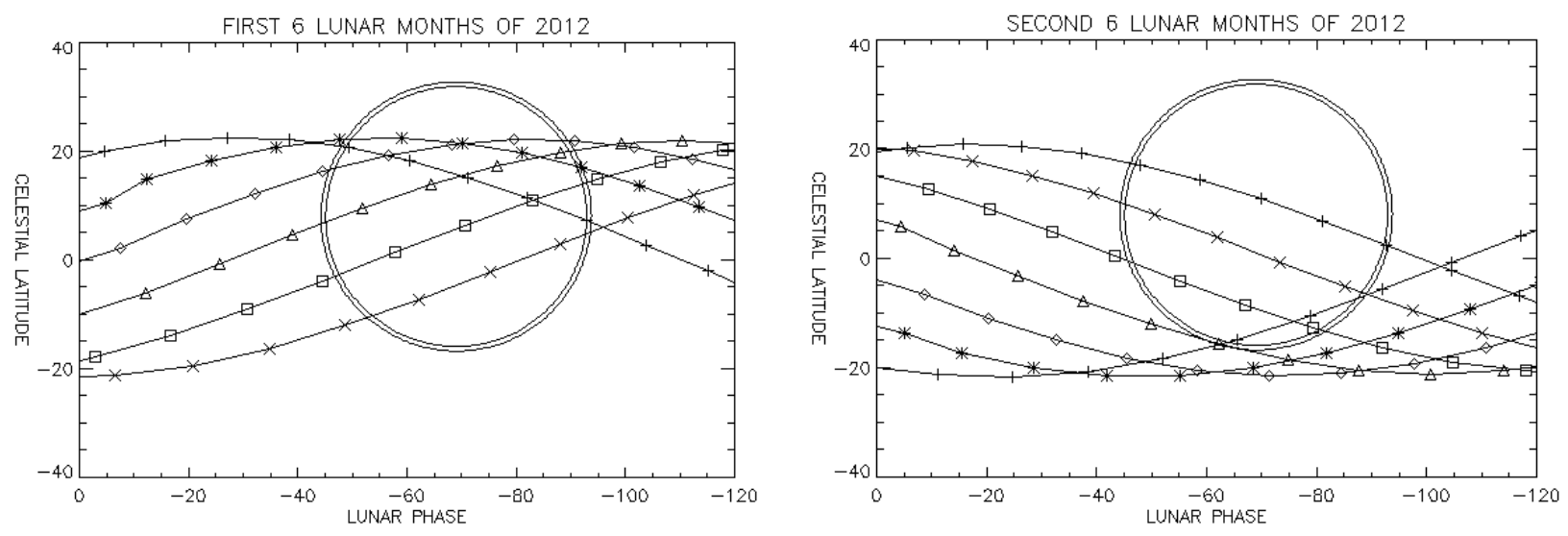

(a)

(b)

Figure 3. VIIRS lunar views in 2012.

a) First six lunar months

b) Second six lunar months. 
As stated above, for the nominal SV trajectory the views are obtained over a large range of phase angles. Even considering only the second view of each month, i.e., with the smaller phase angle, the range is about 44 to 54 degrees. As stated in Ref. 9, the lunar views need to be constrained within a phase range of a few degrees; and to allow cross calibration with MODIS, as close as possible to $-55^{\circ}$.

The lunar phase during the views can be changed by altering the SV trajectory. This is accomplished by a spacecraft roll maneuver. As shown in Figs. 1 and 2, a rotation about the roll axis (out of the page) moves the SV farther from the Earth limb and closer to the negative orbit normal. This reduces the radii of the circles in Fig. 3, which changes the lunar phase at the crossing point. Thus, the observed lunar phase can be selected within a limited range by changing the spacecraft roll angle for each observation. This same approach has been used for MODIS on both Terra and Aqua to maintain close to $-55^{\circ}$ phase for the lunar views. One significant difference between the lunar roll maneuvers performed on Terra and Aqua and those performed on SNPP is the MODIS maneuvers are performed on the dark side of the orbit while the SNPP maneuvers are performed on the sunlit side of the orbit. This is due to a difference in the hardware orientation of the MODIS spaceview port relative to the Terra spacecraft versus the hardware orientation of the VIIRS spaceview sector relative to the SNPP spacecraft.

For VIIRS, an additional constraint is imposed by the thermal keep-out zone (KOZ) for the cryoradiator, which must be protected from any exposure to sunlight. To maintain this constraint with acceptable margin, a limit is imposed on the roll angle for each lunar observation. This also limits the range of phase angles for the lunar views. For the early April and May 2012 lunar views, the phase angle was limited to approximately $-51^{\circ}$ in order to maintain the $\mathrm{KOZ}$ constraint. Since this is also expected to occur in future months and years, it was decided to change the target phase from $-55^{\circ}$ to $-51^{\circ}$ after the April lunar calibration.

Table 1 shows the VIIRS lunar views for 2012. For the first six views, the actual times, roll angles and phases are shown. The remaining entries show the predicted values. As shown, to restrict the range of phase angles and obtain a useful lunar time series, a roll maneuver of between $-10^{\circ}$ and $0^{\circ}$ is required for each lunar view. Much like the overall viewing geometry, these conditions will be essentially the same each year. For the May $31^{\text {st }}$ view, the smallest possible phase angle was nearly $53^{\circ}$; achieving a $51^{\circ}$ phase would have required a positive roll maneuver, moving the SV closer to the Earth horizon.

Table 1. VIIRS lunar observation opportunities in 2012. From June through September, the moon is hidden behind the Earth at the time of the appropriate lunar phase angle.

\begin{tabular}{|c|c|c|c|}
\hline Date & $\begin{array}{c}\text { Time GMT } \\
\text { (H:M:S) }\end{array}$ & $\begin{array}{c}\text { Roll Angle } \\
\text { (deg) }\end{array}$ & $\begin{array}{c}\text { Lunar Phase Angle } \\
\text { (deg) }\end{array}$ \\
\hline 04 Jan & $8: 48: 53$ & -9.49 & 55.41 \\
\hline 03 Feb & $6: 03: 30$ & -5.08 & 55.38 \\
\hline $03 \mathrm{Mar}$ & $23: 59: 22$ & -5.28 & 55.60 \\
\hline $02 \mathrm{Apr}$ & $23: 05: 11$ & -3.99 & 51.24 \\
\hline $02 \mathrm{May}$ & $10: 20: 06$ & -3.23 & 50.92 \\
\hline 31 May & $14: 46: 14$ & 0.00 & 52.96 \\
\hline 25 Oct & $6: 56: 31$ & -4.12 & 51.05 \\
\hline 23 Nov & $21: 15: 54$ & -9.59 & 50.66 \\
\hline 23 Dec & $14: 56: 46$ & -6.96 & 50.92 \\
\hline
\end{tabular}

The VIIRS lunar measurements from the roll maneuvers are compared with values from the USGS lunar model ${ }^{10}$. The VIIRS disk images (irradiances) are compared with the model values for the same viewing conditions, and the results are given as the percent difference of VIIRS from the lunar value. The foundation of the model is the invariance of the lunar surface over periods of multiple millennia or more ${ }^{11}$. In addition, the model accounts for the distances and angles in each VIIRS measurement ${ }^{10}$. The VIIRS comparison with the model is listed in Table 2. The model does not have an absolute calibration, but it can be a common reference for VIIRS and other instruments on-orbit ${ }^{12}$. The relative changes in the VIIRS bands with respect to the Moon are shown in Fig. 4. This is a primary method for determining the deterioration of the radiometric responsivity of the VIIRS bands over time. 
Table 2. Differences of the lunar measurements from the USGS lunar model. The values give the percent differences of the measured lunar irradiances from the model results. The Moon is not an absolute calibration source.

\begin{tabular}{|c|c|c|c|c|c|c|c|}
\hline Date & $\begin{array}{c}\text { Band M1 } \\
412 \mathrm{~nm}\end{array}$ & $\begin{array}{c}\text { Band M2 } \\
445 \mathrm{~nm}\end{array}$ & $\begin{array}{c}\text { Band M3 } \\
488 \mathrm{~nm}\end{array}$ & $\begin{array}{c}\text { Band M4 } \\
555 \mathrm{~nm}\end{array}$ & $\begin{array}{c}\text { Band M5 } \\
662 \mathrm{~nm}\end{array}$ & $\begin{array}{c}\text { Band M6 } \\
646 \mathrm{~nm}\end{array}$ & $\begin{array}{c}\text { Band M6 } \\
865 \mathrm{~nm}\end{array}$ \\
\hline 04 Jan & 3.43 & 5.46 & 10.83 & 11.30 & 12.05 & 12.05 & 11.05 \\
\hline 03 Feb & 2.49 & 5.32 & 10.66 & 11.03 & 10.34 & 8.96 & 5.39 \\
\hline 02 Apr & 2.45 & 5.52 & 11.00 & 11.00 & 8.56 & 5.90 & -0.61 \\
\hline 02 May & 2.53 & 5.53 & 11.00 & 10.65 & 6.65 & 4.33 & -3.26 \\
\hline 31 May & 1.95 & 5.10 & 10.63 & 10.15 & 6.85 & 3.35 & -5.62 \\
\hline
\end{tabular}
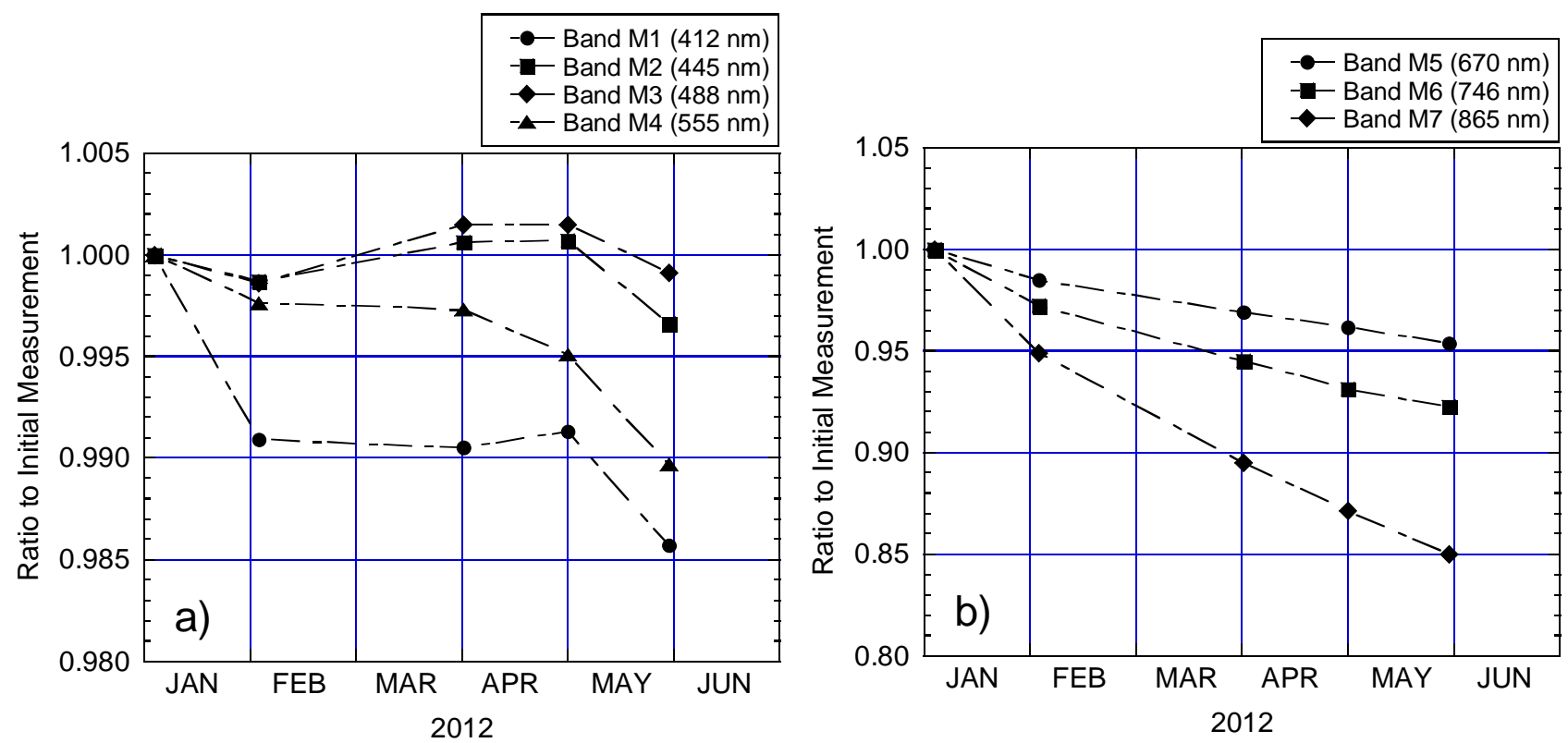

Figure 4. Lunar-based measurement of radiometric sensitivity changes in the VIIRS visible/near-infrared bands. Due to an improper ephemeris calculation that neglected the leap day on 29 February, the 3 Mar lunar roll maneuver was performed a day early, and the measurement opportunity was missed.

a) Measurement results for the VIIRS blue and green bands, 412-nm to 555-nm.

b) Measurement results for the VIIRS red and near-infrared bands, 660-nm to 865-nm

\section{2b. Spacecraft Yaw Maneuvers}

Like its heritage instruments Terra and Aqua MODIS ${ }^{13}$, SNPP VIIRS uses a solar diffuser ${ }^{14}$ to monitor responsivity changes in the visible, near infrared, and short-wave infrared. The MODIS instruments incorporated screens with nominal transmissions of $6.8 \%$ to attenuate the solar irradiance on the diffusers. The laboratory characterizations of the diffusers were made at a limited number of wavelengths and illumination angles ${ }^{15}$, and those characterizations did not include the screens. As a result, system level characterizations of the diffusers and diffuser screens were accomplished on orbit, using the Sun as the illuminating source. Satellite yaw maneuvers, centered on the normal to the diffuser screen, encompassed the entire angular range of solar illumination angles during each MODIS mission. The rotation of the spacecraft in pitch during those measurements covered the angular range in the other direction, allowing two dimensional characterizations of the combined diffuser/screen for the instruments. For each MODIS instrument, there were a total of 16 yaw maneuvers for the characterization ${ }^{5}$. Each yaw maneuver is centered on a solar measurement time approximately $3 \mathrm{~min} 30 \mathrm{sec}$ after the satellite exits the Earth's penumbra into full sunlight. The yaw attitude adjustment is started in darkness and takes $5 \mathrm{~min}$. About $15 \mathrm{sec}$ after the conclusion of the yaw angle adjustment, the spacecraft exits the Earth's umbra followed by the exit from the penumbra $10 \mathrm{sec}$ later. The spacecraft remains in the sunlight at the measurement yaw angle for 6 min $30 \mathrm{sec}$ before the maneuver back to the normal on-orbit yaw angle. This allows 
measurements at all pitch angles for each yaw angle, giving coverage of the full angular range of solar illumination for the mission. As with the initial angle adjustment, the return to the standard yaw angle takes 5 min as well. Figure 5 shows the SNPP spacecraft before, during and after performing the yaw maneuver.

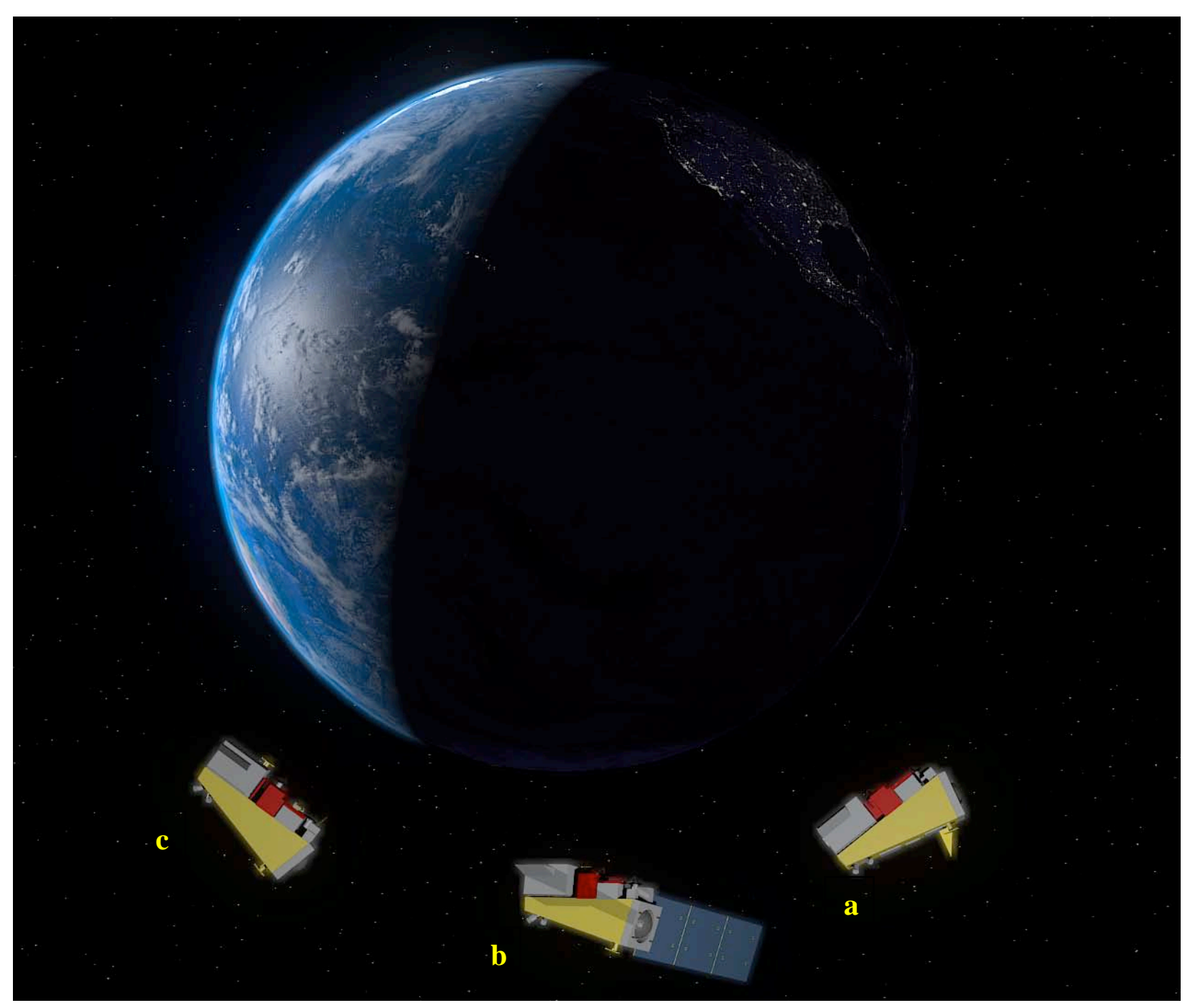

Figure 5. One of a series of 15 SNPP spacecraft yaw maneuvers used to characterize the optical performance of the VIIRS solar diffuser, diffuser screen, and solar diffuser stability monitor. The on-orbit direction of the SNPP spacecraft in this figure is clockwise from right to left.

a. The SNPP spacecraft is in its nominal Earth viewing mode in eclipse.

b. The SNPP spacecraft is yawed to an angle between $-6.0^{\circ}$ and $10.9^{\circ}$ over the South Pole in sunlight but before the Earth terminator. In this position, VIIRS acquires solar diffuser data.

c. The SNPP spacecraft is yawed back to its nominal Earth viewing geometry.

The yaw maneuvers cover solar azimuth angles from $13.6^{\circ}$ to $30.6^{\circ}$ (Table 3 ), the range experienced on orbit. In addition, the maneuvers characterized the angular response of the Solar Diffuser Stability Monitor (SDSM), the on-board ratioing radiometer which measures the change of the diffuser relative to the sun. 
Table 3. Yaw maneuver measurement schedule. The measurements cover the range of azimuth angles on the diffuser found over the course of each year. The maneuvers are referenced to the normal to the diffuser screen, and the azimuth angle is calculated via a transformation.

\begin{tabular}{|c|c|c|c|}
\hline $\begin{array}{c}\text { Date - Time GMT* } \\
\text { (H:M:S) }\end{array}$ & Orbit & $\begin{array}{l}\text { Yaw Angle** } \\
\text { (deg) }\end{array}$ & $\begin{array}{l}\text { Solar Azimuth Angle } \\
\text { (deg) }\end{array}$ \\
\hline 15 Feb - 13:46:44 & 1563 & -6.0 & 13.6 \\
\hline 15 Feb - 15:28:44 & 1564 & -4.6 & 15.0 \\
\hline 15 Feb - 16:09:45 & 1565 & -3.4 & 16.3 \\
\hline 15 Feb - 18:51:15 & 1566 & -2.1 & 16.6 \\
\hline 15 Feb - 20:32:45 & 1566 & -0.8 & 18.9 \\
\hline 15 Feb - 22:14:16 & 1568 & 0.5 & 20.2 \\
\hline 15 Feb - 23:55:46 & 1569 & 1.8 & 21.5 \\
\hline 16 Feb - 01:36:16 & 1560 & 0.0 & 19.6 \\
\hline 16 Feb - 03:18:46 & 1561 & 3.1 & 22.8 \\
\hline $16 \mathrm{Feb}-05: 00: 46$ & 1562 & 4.4 & 24.1 \\
\hline 16 Feb - 06:41:46 & 1563 & 5.6 & 25.4 \\
\hline 16 Feb - 08:23:18 & 1564 & 6.0 & 26.6 \\
\hline 16 Feb - 10:04:48 & 1565 & 8.3 & 28.0 \\
\hline 16 Feb - 11:46:48 & 1566 & 9.6 & 29.3 \\
\hline 16 Feb - 13:26:49 & 1566 & 10.9 & 30.6 \\
\hline
\end{tabular}

*36 seconds after eclipse penumbra exit

**with respect to orbit frame
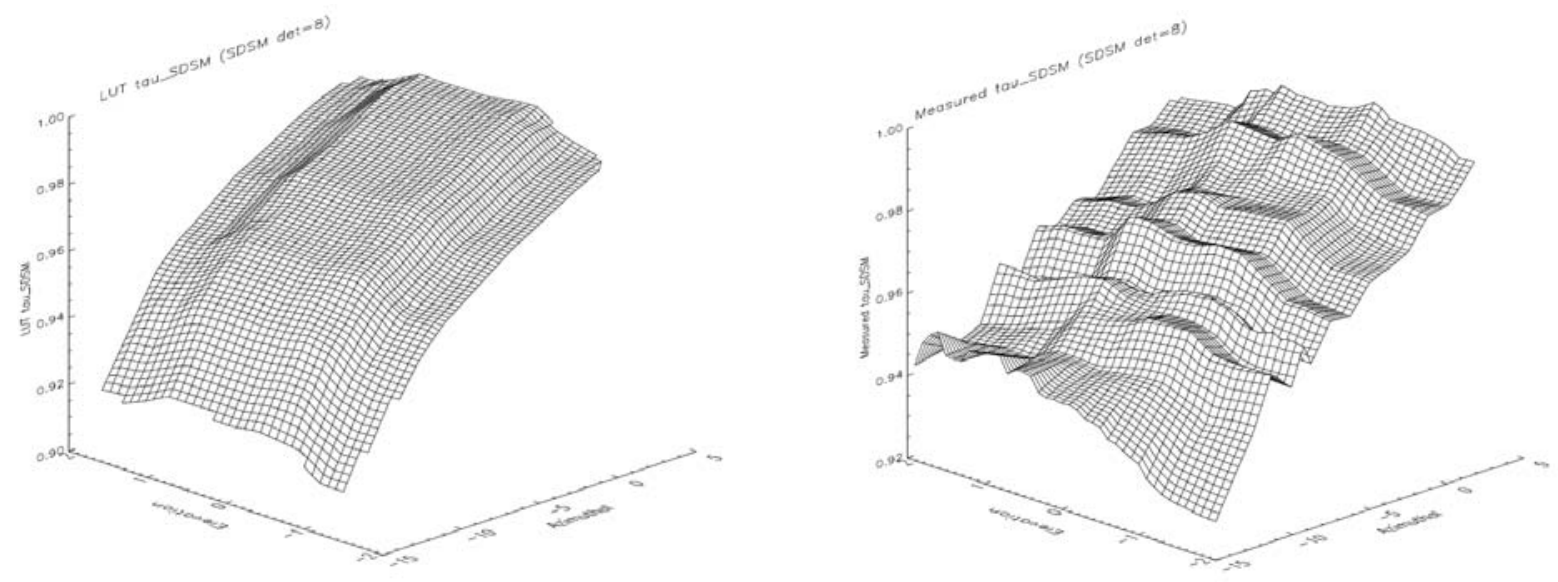

Figure 6. Two dimensional transmission surfaces for the VIIRS SDSM. Both surfaces are normalized to unity at zero azimuth and zero elevation, The left-hand panel gives the result from the prelaunch measurements. The right-hand panel gives the results from the on orbit yaw maneuvers.

In Figure 6, the SDSM solar view screen transmissions derived from the pre-launch measurements (left plot) and from the on-orbit yaw maneuver data (right plot) are shown for SDSM detector 8. The pre-launch transmission was sparsely measured in both SDSM azimuth and elevation. The screen transmission derived on-orbit revealed features unresolved by the pre-launch measurement; in particular ridges in SDSM elevation at roughly constant SDSM azimuth are observed. The on-orbit screen transmission was constructed from all good quality yaw maneuver data within an SDSM elevation of $\pm 2^{\circ}$ (no restriction is made in SDSM azimuth). 

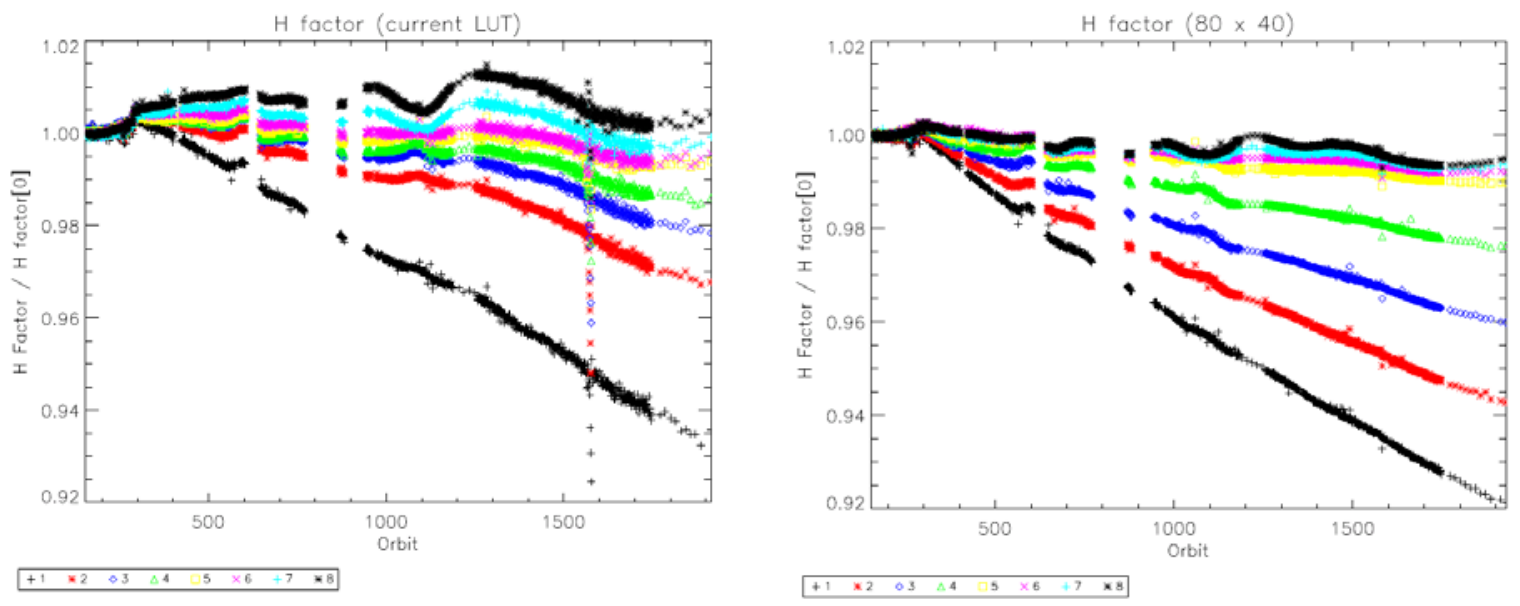

Figure 7. Yaw angle results for the Solar Diffuser Stability Monitor bands M1 through M8 (412-nm to 1260-nm). The left hand panel gives the diffuser change ( $\mathrm{H}$ factor) over 1900 orbits derived from the prelaunch measurements. The right hand panel gives the on-orbit diffuser change using the results from the yaw maneuvers.

Although one would expect a pinhole attenuation screen transmission to be achromatic, the derived screen transmission also accounts for differences in path between detectors with the SDSM integrating sphere; as a result, the SDSM solar screen transmission constructed here is detector dependent.

The $\mathrm{H}$ factor (a measure of the solar diffuser BRDF degradation due to UV exposure) is plotted in Figure 7 using the pre-launch (left plot) and yaw maneuver derived (right plot) SDSM solar screen transmissions. The use of the on-orbit transmission significantly reduced the variation in the $\mathrm{H}$ factor (due to improved resolution of the fine structure in the screen transmission as seen in Figure 6). The updated results are more consistent with MODIS experience.

\section{2c. Spacecraft Pitch Maneuvers}

Because it operates at near room temperature, the VIIRS instrument is a source of emitted radiation for the thermal wavelengths. As a result, the VIIRS thermal detectors measure both the Earth radiance and a significant instrument-generated radiance. The instrument-generated thermal radiance varies slowly over time and is nearly constant over the period of a single rotation of the mirror. The instrument radiance offset is present in measurements of the calibration radiance from the onboard blackbody plus the zero radiance from cold space, both of which are made with every rotation of the mirror. In addition to reflecting the incident radiance, the mirror serves as a source of emitted radiance as well. For the mirror, the emissivity $(\varepsilon)$ and the reflectance $(\rho)$ sum to unity. In addition, the reflectance is a function of the angle of incidence of the Earth radiance upon it. This effect is called the response versus scan angle (RVS). It varies over each rotation of the mirror and cannot be subtracted as a constant offset.

For the Terra MODIS instrument, pre-launch measurement limitations precluded the production of reliable systemlevel RVS data sets for the thermal bands ${ }^{16}$. This caused striping and banding in the on-orbit measurements. A nominal RVS correction was used at launch, and an improved RVS characterization was performed using on-orbit measurements. For these measurements, the Terra spacecraft performed a back flip on the nighttime portion of the orbit. This allowed MODIS to collect a set of scans of cold space, instead of the warm Earth. For these scans, there was no incident radiance upon the mirror and the change in the instrument output over each scan came from change in the emissivity of the mirror with scan angle. When normalized to the output at the angle for the deep space view (zero offset), these data allowed the calculation of the relative change in the mirror's emissivity and reflectance over each scan. 


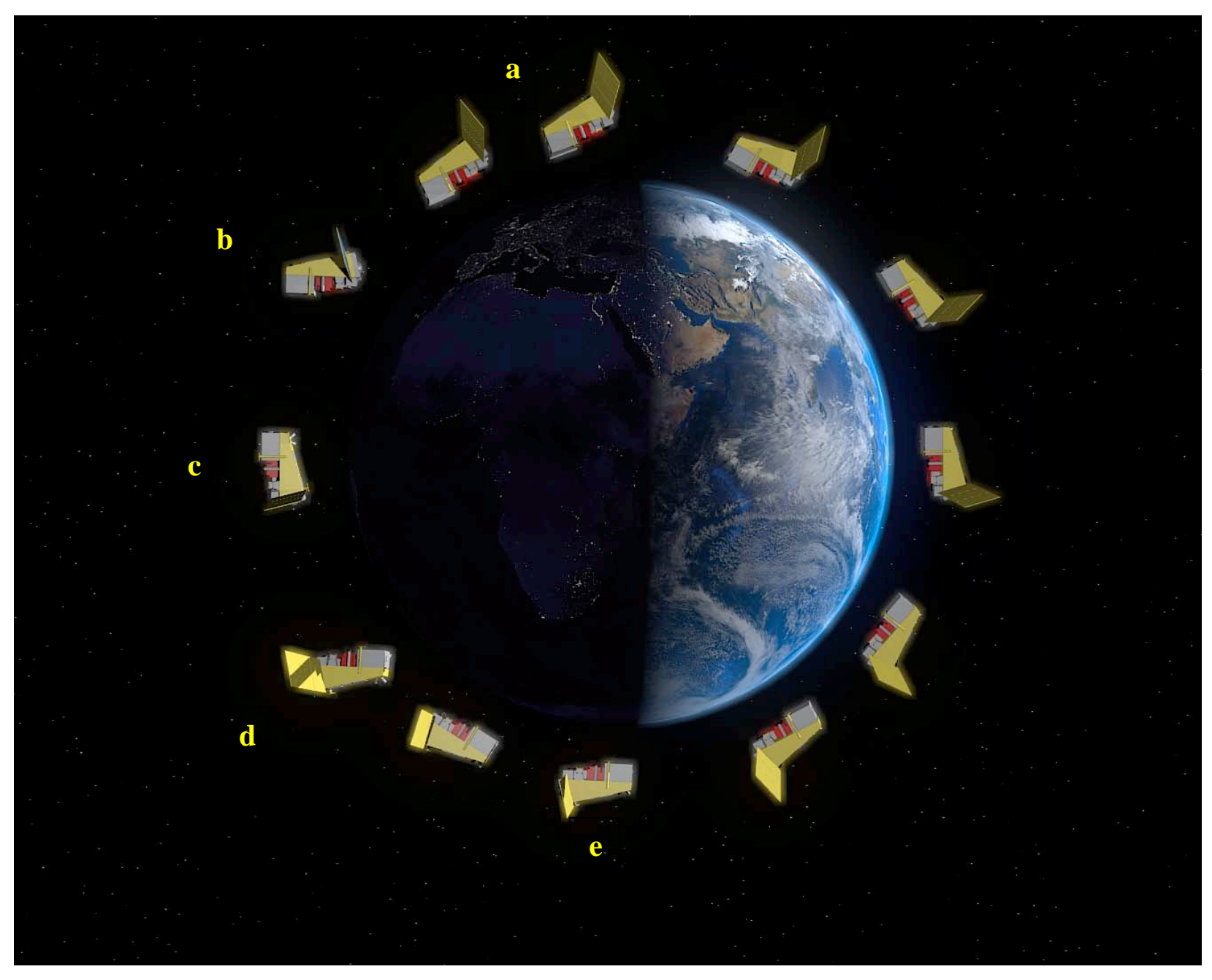

Figure 8. The SNPP spacecraft pitch-over maneuver enabling VIIRS to scan deep space. Motion of the SNPP spacecraft is counterclockwise in this figure. On the sunlit portion of the orbit, the VIIRS instrument is in its nominal Earth viewing geometry with the spacecraft in its ascending orbit.

a. The maneuver begins with a slight pitch down of the nose of the SNPP spacecraft.

b. The nose of the SNPP spacecraft in the process of pitching up.

c. The SNPP spacecraft is pitched completely away from viewing the Earth on the dark side of the orbit, and the VIIRS instrument is oriented to view deep space.

d. The pitch maneuver continues to return the SNPP spacecraft to nominal Earth viewing mode.

e. The SNPP spacecraft has returned to its nominal Earth viewing geometry.

In the SNPP VIIRS instrument, a half angle mirror (HAM) is used to compensate image rotation caused by the Rotating Telescope Assembly (RTA). During VIIRS operation, the angle of incidence of remotely sensed photons onto the HAM changes as the RTA is rotated. In order to measure the RVS of the HAM, a pitch maneuver of the SNPP spacecraft was performed on the dark side of orbit 1636 on 20 Feb 2012. It was accomplished using a constant rate back flip of the spacecraft over a period of $33 \mathrm{~min} 45 \mathrm{sec}$. For $5 \mathrm{~min}$ before the entrance of the spacecraft into the Earth's full shadow (eclipse umbra), the speed of the pitch wheels was adjusted to give the spacecraft a constant pitch rate of 0.166 deg $\sec ^{-1}$. At eclipse umbra exit, the speed of the pitch wheels was again adjusted over a 5 min period to return the spacecraft to constant Earth pointing. Figure 8 shows the orientation of the SNPP instrument at several positions during the pitch over maneuver. 

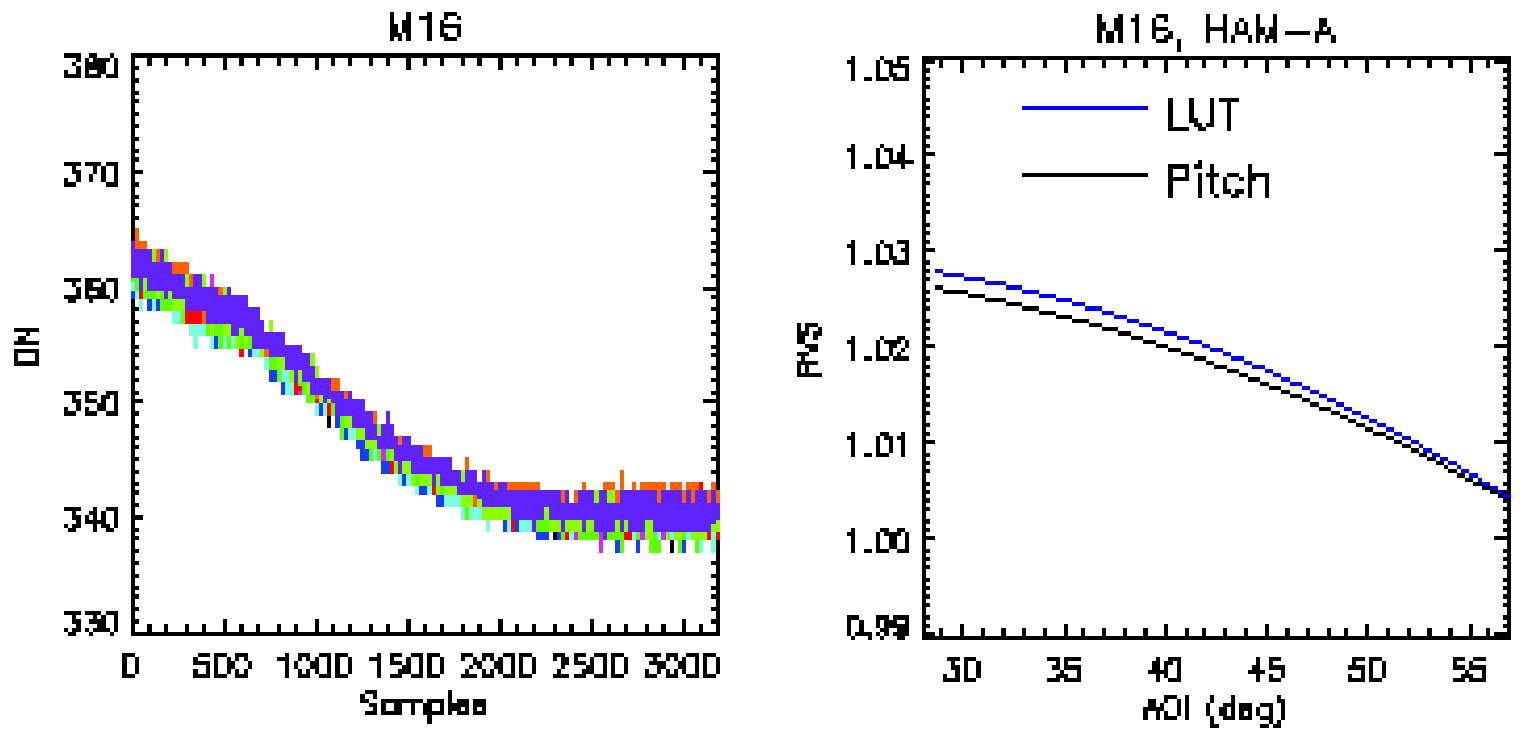

Figure 9. Results of pitch maneuver for VIIRS band M16. The left hand panel shows the set of deep space measurements across the sweep of the RTA and HAM. The right hand panel shows the derived response versus scan angle versus the angle of incidence on side A of the two-sided HAM from the pre-launch measurements (LUT) and from the pitch maneuver (Pitch).

The pitch maneuver started at 18:15:34 GMT and ended at 18:59:19 GMT on Feb 20, 2012. Since a complete view of deep space occurred at the middle point of the maneuver, one might be expected to use data collected around 18:36:00 GMT. However, at around 18:30:00 GMT, temperatures of the cold focal planes started to rise rapidly as a result of the thermal infrared absorption of the Earth's radiation due to the pitch maneuver. Thus, data collected slightly earlier than 18:30:00 GMT is selected to avoid any possible impact due to the rapid rise in the cold focal plane temperatures. A total of 40 consecutive scans ( 20 for each HAM side) of data samples are collected from the pitch maneuver. Each scan covers the entire width of the Earth view with 3200 pixels for M bands with exception of 6304 pixels for M13 and 6400 pixels for I bands. The left hand plots in Fig. 9 gives the raw response versus sample number based on multiple scans at HAM-A side for band M16. The fact that the response is not flat crossing the entire angular range indicates that the reflectivity of the scan mirror changes with the scan angle. Thus, use of the data collected from the pitch maneuver can provide useful information to assess the response versus scan angle (RVS). Comparison of the pitch maneuver and prelaunch RVS results (see the right hand panel of Fig. 9) shows an agreement of within $0.4 \%$ on average for all thermal emissive bands (TEB), which is generally within the requirement of $0.3 \%$ allowed for the characterization of the prelaunch RVS. The result of this study indicates that the prelaunch RVS is well characterized and there is no need to make any adjustment for current on-orbit use.

\section{CONCLUSIONS}

The pioneering, successful use of monthly lunar views by the SeaWiFS instrument ${ }^{1}$ and subsequent use by a number of EOS instruments have established the Moon as an important, stable on-orbit calibration target. Using negative small angle rolls of the SNPP spacecraft, the VIIRS instrument has acquired images of a $-51^{\circ}$ to $-55^{\circ}$ Moon every month it has been available since launch. The SNPP VIIRS instrument, like the MODIS Terra and Aqua instruments before it, employs a Spectralon solar diffuser for on-orbit radiometric calibration of its reflected solar bands. Changes in the solar diffuser spectral bidirectional reflectance are monitored using a Solar Diffuser Stability Monitor (SDSM). The SDSM is effectively a multiband filter ratioing radiometer which views the Sun directly and the solar illuminated diffuser. As shown in Fig. 10, VIIRS measurements of integrated lunar irradiance complement the measurements of the Solar Diffuser Stability Monitor (SDSM) on-board the VIIRS instrument and validate those measurements to $1 \%$ or better. Although not addressed in detail in this paper, the Moon can also be used as an on-orbit cross-comparison target between instruments ${ }^{12}$. For this reason, VIIRS measurements of the Moon at lunar phases between $-51^{\circ}$ and $-55^{\circ}$ were designed 
to match as closely as possible the lunar phase viewed by the MODIS Aqua instruments. The Moon can also be used as a target for spatial characterization ${ }^{4}$.

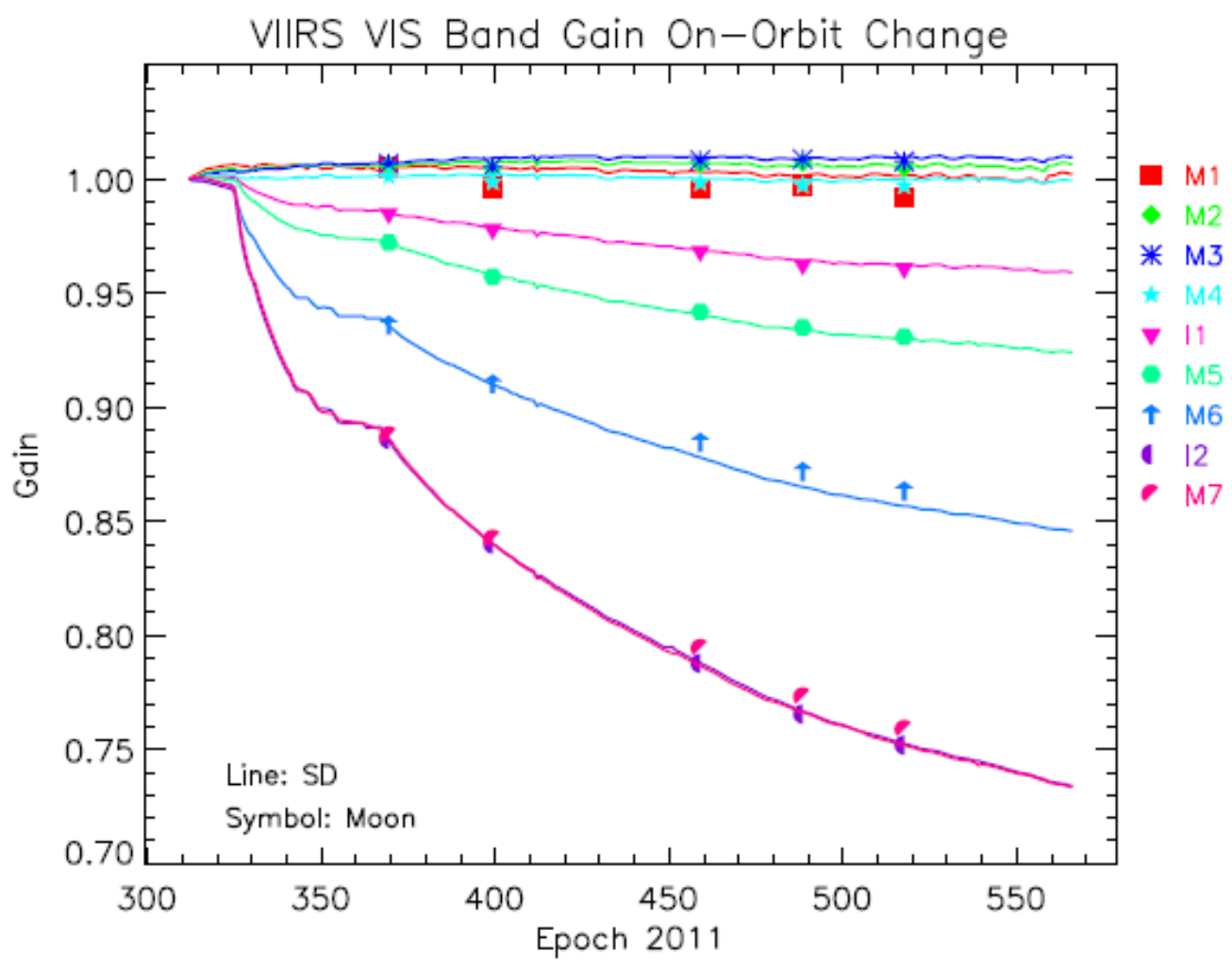

Figure 10. On-orbit radiometric responsivity trending of the VIIRS visible and near infrared bands using the solar diffuser stability monitor (SDSM) and lunar images. The VIIRS band wavelengths in the legend are as follows: M1: $412 \mathrm{~nm}$; M2: $445 \mathrm{~nm}$; M3: 488 nm; M4: 555 nm; M5: 662 nm; M6: 646 nm; M6: 865 nm.

The challenge experienced in understanding the optical performance of the solar diffuser screen and solar diffuser on MODIS Terra and Aqua ${ }^{17,18}$ strongly supported performing the set of on-orbit yaw maneuvers on VIIRS. The number and angular range of yaw angles comprising these 15 maneuvers performed on successive orbits were based on the predicted range of solar incident angles onto the VIIRS diffuser over a season. The data obtained from these maneuvers was used to update and improve the solar diffuser look-up-table entries, which previously were determined not at solar diffuser, solar diffuser screen, SDSM subsystem level.

The pitch over maneuver of the SNPP spacecraft enabled VIIRS to characterize the response versus scan angle of its HAM particularly for the thermal infrared bands. This maneuver, with direct heritage to the EOS Terra spacecraft which performed the identical maneuver twice, benefited not only VIIRS but also the CERES and ATMS instruments. For CERES, the pitch maneuver enabled the direct determination of the dc offsets of the series instrument under zero g conditions. The pitch maneuver enabled the determination of the ATMS instrument sidelobes in the track direction and the presence of any contamination signal into those sidelobes from surrounding instruments or spacecraft structures.

The performance of on-orbit spacecraft maneuvers in support of the calibration and characterization of the SNPP instruments required clear descriptions of and rationales for the required maneuvers from the SNPP science community early in the project. That way, the capability of the spacecraft to perform these maneuvers was included in its design specification. Thermal, mechanical, and optical impacts not only to the spacecraft but also to all on-board instruments were fully studied and understood well before launch. A capable and professional SNPP Mission Operations Team (MOT) performed each spacecraft maneuver flawlessly. 


\section{REFERENCES}

1. Eplee, R.E., R.A. Barnes, F.S. Patt, G. Meister, and C.R. McClain, "SeaWiFS lunar calibration methodology after six years on orbit," in Earth Observing Systems IX, W.L. Barnes and J.J. Butler, eds., Proc. SPIE, 5542, 1-13, 2004.

2. Kieffer, H.H, P. Jarecke, and J. Pearlman, "Initial lunar calibration observations by the EO-1 Hyperion imaging spectrometer," in Imaging Spectrometry VII, M.R. Descour and S.S. Shen, eds., Proc. SPIE, 4480, 246-258, 2002.

3. Sun, J.-Q., X. Xiong, W.L. Barnes, and B. Guenther, "MODIS reflective solar bands on-orbit lunar calibration," IEEE Trans. Geosci. Remote Sens., 45, 2382-2393, 2006.

4. Xiong, X., J. Sun, S. Xiong, and W.L. Barnes, "Using the moon for MODIS on-orbit spatial characterization,” in Sensors, Systems, and Next-Generation Satellites VI, J.B. Lurie, M.L. Aten, and H. Shimoda, eds., Proc. SPIE, 5234, 480-486, 2004.

5. Xie, X., X. Xiong, D. Moyer, J. Sun, X. Liu, and W. Barnes, "Analysis of MODIS solar diffuser screen vignetting function,” in Earth Observing Systems X, J.J. Butler, ed., Proc. SPIE, 5882, 58820T, 2005.

6. Wang, Z. and X. Xiong, "Characterization of MODIS SD screen vignetting function using observations from spacecraft yaw maneuvers,” in Earth Observing Systems XIV, J.J. Butler, X. Xiong, and X. Gu, eds., Proc. SPIE, 6452, 645216, 2009.

7. Kleespies, T.J., R. Smith-Dearring, J. Woodward, J. Shepherd, C. Gliniak, W. Chadwick, J. Walters, and D. Han, "Evaluation of scan asymmetry in the NOAA-14 Microwave Sounding Unit by a pitch maneuver," IEEE Geosci. Remote Sens. Lett., 4, 621-623, 2006.

8. Sun, J.-Q. and X. Xiong, "Solar and lunar observation planning for Earth-observing sensor," in Sensors, Systems, and Next-Generation Satellites XV, R. Meynart, S.P. Neeck, and H. Shimoda, eds. Proc. SPIE, 8166, 816610, 2011.

9. Patt, F.S., R.E. Eplee, R.A. Barnes, G. Meister, and J.J. Butler, "Use of the Moon as a calibration reference for NPP VIIRS,” in Earth Observing Systems X, J.J. Butler, ed., Proc. SPIE, 5882, 588215, 2005.

10. Kieffer, H.H., and T.S. Stone, "The spectral irradiance of the Moon,” Astron. J., 129, 2886-2901, 2005.

11. Kieffer, H.H., "Photometric stability of the lunar surface," Icarus, 130, 323-326, 1996.

12. Eplee, R.E. Jr., J.-Q. Sun, G. Meister, F.S. Patt, X. Xiong, and C.R. McClain, "Cross calibration of SeaWiFS and MODIS using on-orbit observations of the Moon," Appl. Opt. 50, 120-133, 2011.

13. Barnes, W.L., T.S. Pagano, and V.V. Salomonson, "Pre-launch characteristics of the Moderate Resolution Imaging Spectroradiometer (MODIS) on EOS AM-1,” IEEE Trans. Geosci. Remote Sens., 36, 1088-1100, 1998.

14. Schueler, C., J.E. Clement, P.Ardanuy, C. Welsh, F. DeLuccia, and H. Swenson, "NPOESS VIIRS sensor design overview,” in Earth Observing Systems VI, W.L. Barnes, ed., Proc. SPIE, 4483, 11-23, 2002.

15. Pavlov, M., M. Byers, and J.A. Walker, "Bidirectional reflectance factor (BRF) characterization of the MODIS flight solar diffuser,” in Earth Observing Systems III, W.L. Barnes, ed., Proc. SPIE, 3439, 256-268, 1998.

16. Xiong, X., V. Salomonson, K. Chiang, A. Wu, B. Guenther, and W. Barnes, "On-orbit characterization of RVS for MODIS thermal emissive bands," in Passive Remote Sensing of the Atmosphere and Clouds IV, S.C. Tsay, T. Yokota, and M-H Ahn, eds., Proc. SPIE, 5652, 210-218, 2004.

17. Waluschka, E., J. Esposito, J. Sun, X. Wang, and X. Xiong, "MODIS solar diffuser-modeled and actual performance,” in Earth Observing Systems VI, W.L. Barnes, ed., Proc. SPIE, 4483, 146-156, 2002.

18. Waluschka, E., X. Xiong, D. Moyer, B. Guenther, W. Barnes, and V.V. Salomonson, “ MODIS solar calibration screen modeling," in Passive Optical Remote Sensing of the Atmosphere and Clouds VI, S.C. Tsay, T. Tokota, and M-H Ahn, eds., Proc. SPIE, 5652, 189-199, 2004. 